\title{
Cardiac surgery is associated with biomarker evidence of neuronal damage
}

Marek Alifier ${ }^{1}, \mathrm{MD}, \mathrm{PhD}$, Bob Olsson ${ }^{2,3}, \mathrm{MD}, \mathrm{PhD}$, Ulf Andreasson ${ }^{3}, \mathrm{PhD}$, Nicholas C. Cullen $^{2,4}$, Jolanta Czyżewska ${ }^{5}$, PhD, Piotr Jakubów ${ }^{6}$, MD, Andrzej Sieśkiewicz ${ }^{7}$, MD, Anna Stasiak-Barmuta $^{1}$, Prof. Dr. med, Tomasz Hirnle ${ }^{6}$, MD, Johannes Kornhuber ${ }^{8}$, Prof. Dr. med, Henrik Zetterberg ${ }^{2,3,9,10}$, MD, PhD, Piotr Lewczuk ${ }^{8,11}$, Prof. Dr. med, and Kaj Blennow ${ }^{2,3}$, $\mathrm{MD}, \mathrm{PhD}$.

${ }^{1}$ Department of Clinical Immunology, Medical University of Bialystok Clinical Hospital, Poland, ${ }^{2}$ Department of Psychiatry and Neurochemistry, Sahlgrenska Academy at the University of Gothenburg, Mölndal, Sweden. ${ }^{3}$ Clinical Neurochemistry Laboratory, Sahlgrenska University Hospital, Mölndal, Sweden, ${ }^{4}$ Department of Psychiatry, Perelman School of Medicine, University of Pennsylvania, Philadelphia, PA, USA. ${ }^{5}$ Department of Clinical Laboratory Diagnostics, Medical University of Bialystok Clinical Hospital, Poland, ${ }^{6}$ Department of Cardiosurgery, Medical University of Bialystok Clinical Hospital, Poland, ${ }^{7}$ Department of Otolaryngology, Medical University of Bialystok Clinical Hospital, Poland, ${ }^{8}$ Department of Psychiatry and Psychotherapy, Universitätsklinikum Erlangen, and FriedrichAlexander-Universität Erlangen-Nürnberg, Erlangen, Germany, ${ }^{9}$ Department of Neurodegenerative Disease, UCL Institute of Neurology, London, UK, ${ }^{10}$ UK Dementia Research Institute at UCL, London, UK, ${ }^{11}$ Department of Neurodegeneration Diagnostics, Medical University of Bialystok Clinical Hospital, Bialystok, Poland.

Abstract word count: 194, word count: 3402, References: 29, Figures 3, Tables 3.

Keywords: Cardiac surgery, extracorporeal circulation, NFL, tau, plasma.

Abbreviated title: Neuronal damage in cardiac surgery 
Corresponding author: Bob Olsson, $\mathrm{MD}, \mathrm{PhD}$, Department of Psychiatry and Neurochemistry, University of Gothenburg, V-house, Sahlgrenska University Hospital Mölndal, 43180 Mölndal, Sweden, Phone, +46 3134324 00, Fax + 4631343 24 26, E-mail: bob.olsson@neuro.gu.se. 


\begin{abstract}
BACKGROUND: Anesthesia and surgery is commonly associated with central nervous system sequelae and cognitive symptoms, which may be caused by neuronal injury. Neuronal injury can be monitored by plasma concentrations of the neuronal biomarkers tau and neurofilament light protein (NFL). Currently, there are no studies examining whether neuronal injury varies between surgical procedures.
\end{abstract}

OBJECTIVE: Our aim was to investigate if neuronal damage is more frequent after cardiac than after otolaryngeal surgery, as estimated by tau and NFL concentrations in plasma.

METHODS: Blood samples were drawn before, during and after surgery and concentrations of tau, NFL, A $\beta 40$ and $A \beta 42$ were measured in 25 patients undergoing cardiac surgery ( 9 offpump and 16 on-pump) and 26 patients undergoing otolaryngeal surgery.

RESULTS: Tau increased during surgery $(1752 \%, \mathrm{P}=0.0001)$ and NFL rose seven days postsurgery $(1090 \%, \mathrm{P}<0.0001)$ in patients undergoing cardiac surgery; even more in patients onpump than off-pump. No changes were observed in patients undergoing otolaryngeal surgery and only minor fluctuations were observed for $\mathrm{A} \beta 40$ and $\mathrm{A} \beta 42$.

CONCLUSION: Cardiac surgery is associated with neuronal injury, which is aggravated by extracorporeal circulation. Analyses of NFL and tau in blood may guide development of surgical procedures to minimize neuronal damage. 


\section{Introduction}

Initially, it was thought that once the post-op period passed, any effects by anaesthesia and surgery on cognition would be reversed. However, several studies have shown that problems with cognition are common after surgery and this was already acknowledged in the 1960s [1, 2]. Post-operative cognitive dysfunction (POCD) is frequent after general surgery [3] and possibly even more so after cardiac surgery [4]. Research into POCD has focused on causes external to the CNS such as physiological perturbations including hypoxemia, hypotension [3], microembolism [5], or inflammation [6]. Clinical studies have also tried to evaluate the effect of anaesthesia, by comparing regional versus general anaesthesia but only found no or minor effect [7-9].

It has been suggested that cognitive problems after anaesthesia and surgery may be caused by neuronal damage. One study investigating this found small and transient changes in the markers NSE and S100B after cardiac surgery [10], but neither of these markers are specific to CNS injury [11]. Other markers which better reflect neuronal damage include the microtubule-tubule associated protein tau and the neurofilament light protein (NFL). Tau is a protein specifically expressed in neurons and is currently used as a clinical biomarker primarily of cortical neuronal damage [12], but is also very sensitive to acute neuronal injury $[11,13]$. NFL together with its medium and heavy counterpart make up the intermediate axonal filaments called neurofilaments that determine axonal calibre and in part axonal velocity [14]. NFL is also a well-established biomarker for acute neuronal damage, both in cerebrospinal fluid (CSF) and in blood $[11,15]$. Both tau and NFL can be analysed in blood by sensitive analyses $[16,17]$. 
Recently, Evered et al found that tau increased $250 \%$ and NFL increased $67 \%$ during anaesthesia and surgery indicating neuronal injury [18]. However, since that cohort was mixed it is presently unclear whether neuronal injury is more common in any type of surgery. To address this we collected samples before, during and after cardiac surgery and otolaryngeal surgery and measured plasma tau and NFL to test the hypothesis that surgery in general and cardiac surgery in particular is associated with neuronal injury reflected by changes in these biomarkers. Furthermore, we compared cardiac surgery patients on and off pump to investigate the impact of extracorporeal circulation on neuronal injury. We also measured plasma levels of the $\mathrm{AD}$ markers $\mathrm{A} \beta 40$, which is thought to reflect the individual production of $A \beta$ with normal levels in Alzheimer's disease (AD) [12], and A $\beta 42$ which in CSF is an AD biomarker with lower levels due to deposition into amyloid plaques $[12,19]$, to test that there is no general, unspecific release of brain-specific proteins by general anaesthesia and surgery. We also measured NFL and tau in patients with myocardial infarction to rule out that these biomarkers are released from the myocardium during damage.

\section{Material and methods}

\section{Study subjects undergoing cardiac or otolaryngeal surgery}

The study consisted of 26 patients who underwent otolaryngeal surgery and 25 subjects who underwent cardiac surgery at the University Teaching Hospital, Bialystok, Poland (Tables 1 and 2). The study was approved by the Ethical Committee, Medical University of Bialystok Clinical Hospital, Poland, and all patients gave informed consent.

Inclusion criteria included an ejection fraction (EF) above $40 \%$ and that the one and the same anesthesiologist was scheduled for the planned surgery. Exclusion criteria were type 1 diabetes, non-stable endocrine disease or thyrotoxicosis, active infectious disease, a severely 
impaired immunological function (i.e. post-transplantation, autoimmune disease or cancer treatment), severe psychiatric illness, severe depression, or acute surgical procedure.

Blood samples were taken from the radial artery at baseline (sample 1), during surgery (sample 2), at the end of surgery (sample 3), 24 hours post-surgery (sample 4), and 7 days post-surgery (sample 5) and collected into standardized polypropylene collection test tubes containing EDTA. Within 30 minutes the blood was centrifuged (1200 g, $5 \mathrm{~min}$ ) and plasma was aliquoted and frozen at $-80 \mathrm{C}$.

\section{Subjects sampled during myocardial infarction}

Blood samples from sixteen individuals (7 females and 9 men; age range 45-89) with myocardial infarction were collected at the Sahlgrenska University Hospital in Mölndal.

\section{Anaesthesia and surgery}

Oral benzodiazepine (midazolam, 0,2. $\mathrm{mg} / \mathrm{kg}$ ) was used as premedication. All patients undergoing either cardiac or otolaryngeal surgery were given general anesthesia with the same set of pharmacological agents: sulfentanil, pancuronium and etomidate as induction and sevoflurane as maintenance anesthesia. The concentrations of respiratory gases (oxygen, air, and sevoflurane) were continuously monitored by real-time spectroscopy. The depth of sevoflurane anesthesia was assessed by clinical and hemodynamic parameters and drug concentrations in the respiratory mixture was measured spectrophotometrically by gas analyzer and adjusted to the level of minimum alveolar concentration (MAC), calculated with the use of algorithm for Dräger Optima. MAC was maintained between 1.1-1.5. Normal arterial blood gas values were secured by mechanical ventilation. $\mathrm{pH}$ was maintained between 7.43 and 7.45. Arterial acid-base balance assessment was performed according to the calculated Siemens algorithm for Siemens Rapid Lab 348. During the extra-corporeal circulation, the gasometric values were recalculated according to the alpha-stat algorithm. All 
patients with extracorporeal circulation had gas values assessed every 15-30 minutes; patients without extra-corporeal circulation - every 30 minutes. $\mathrm{CO}_{2}$ was assessed by capnography at the same time points and were maintained between 30-40 mmHg. Multi-parameter ECG with ST segment and rhythm disturbance analysis of five precordial leads was recorded. Measurements of carbon dioxide by capnometry plethysmography, heart rate, hemoglobin saturation were also monitored.

Peripheral blood pressure measurements were performed using continuous invasive measurements with a canula inserted into right radial artery in all patients. In both study groups, central venous pressure (CVP) were measured in vena cava superior (VCS) using a canula inserted to VCS via the right internal jugular vein. In addition, patients undergoing valvular surgery, had a Swan-Ganz catheter (Braun AG) inserted in a small pulmonary artery that also measured blood pressure, pulmonary artery pressure, wedge pressure and calculated cardiac projection by thermodilution.

CABG was performed at normothermia and AVR at 32 degrees Celsius. Cardioplegic and potassium solutions were used according to the method of Calafiore [20] with warm cardioplegia for $\mathrm{CABG}$ procedures.

Extracorporeal circulation was carried out on a Stockert camera using Medtronic Fusion oxygenators. The flow of respiratory gases was calculated from a rotameter (Sechrist, Air/Oxygen gas mixers Model 20090, USA). Standard doses of unfractionated heparin (UHF) were used as an anticoagulant during extracorporeal circulation with doses calculated according to body weight and antithrombin coagulation time (ACT).

Patients prophylactically received two doses of intraoperative cefazolin and low molecular weight heparin and the patients undergoing coronary surgery also received aspirin. 
To minimize blood loss and to improve surgical conditions mean systemic arterial pressure was maintained between 60 to $70 \mathrm{mmHg}$ in patients undergoing cardiac and otolaryngeal surgery alike.

\section{Biomarker analysis}

Plasma levels of $A \beta 40$ and $A \beta 42$ were measured with a multiplex assay from Fujirebio (Gent, Belgium) on Luminex IS100 and plasma levels of tau and NFL were measured with an ultrasensitive single molecule array (Simoa, Lexington, MA) technology, as previously described $[16,17]$. Troponin $\mathrm{T}$ concentration was measured in clinical routine (Roche, Diagnostics, Vienna, Austria). All plasma levels were corrected for IgG levels to compensate for possible differences in plasma volume due to fluid replacement during surgery. This did not change results or significances as compared with uncorrected values (data not shown). Biomarker measurements were performed in clinical laboratory using methods and procedures accredited by the Swedish Board for Accreditation and Conformity Assessment (SWEDAC). The measurements were performed by board-certified laboratory technicians who were blinded to clinical data.

\section{Statistical analysis}

Analysis of differences in neuronal protein levels was performed using linear mixed effects models, with procedure and time included as fixed effects and participants as a random effect. In order to better compare plasma biomarker levels between patients undergoing the two different forms of surgery, we normalized the values by setting baseline (i.e. before surgery) levels to $100 \%$, and dividing concentrations in longitudinal samples with the baseline value in each patient (raw data values are given Table 3). We included age and sex as covariates and evaluated main and interaction effects using the Wald test. Comparisons between-time/withinprocedure (difference between time points within the cardiac surgery or the otolaryngeal 
surgery group) and within-time/between-procedure (difference between the cardiac surgery and the otolaryngeal surgery group at a specific time point) were evaluated by contrasting least-square means. We report an effect size for each comparison as the percent difference in neuronal protein levels between compared groups as estimated by the least-square means, along with $\mathrm{P}$ values adjusted for multiple comparisons using the Tukey method.

Associations between neuronal protein levels and troponin $\mathrm{T}$ levels in myocardial infarction patients were calculated using partial Spearman's correlation adjusted for age and sex.

All tests were pre-planned and two-sided. Statistical analysis was performed using the $\mathrm{R}$ programming language, version 3.4.3, along with the nlme, version 3.1, and lsmeans, version 2.27, packages.

\section{Results}

\section{The effect of surgery on serum levels of tau}

Six out of $200(3 \%)$ measurements were missing for tau due to lack of sample volume. Tau levels were significantly elevated in the patients undergoing cardiac surgery both during (mean difference, 1701\%; $\mathrm{P}=0.0001$ ) and at the end of surgery compared to baseline (mean difference, $1752 \%$; $\mathrm{P}<0.0001$; Figure 1A). However, there were no significant change in tau levels over time in the patients undergoing otolaryngeal surgery. Tau levels were significantly increased during and at the end of surgery in patients undergoing cardiac surgery compared to the same time points in otolaryngeal surgery (mean difference, 1413\%;, P=0.004; mean difference, 1416\%; P=0.004, respectively, Figure 1A).

\section{The effect of surgery on serum levels of NFL}

Six out of 200 (3\%) measurements were also missing for NFL due to lack of sample volume. NFL levels in the cardiac surgery group seven days post-surgery were significantly increased compared to all other time points - baseline (mean difference, 1090\%; $\mathrm{P}<0.0001$ ), during 
surgery (mean difference, 1042\%; $\mathrm{P}<0.0001$ ), at the end of surgery (mean difference, 991\%; $\mathrm{P}<0.0001$ ), and 24 hours post-surgery (mean difference, 933\%; $\mathrm{P}<0.0001$, Figure 1B). As for tau, there were no significant differences over time for NFL in the otolaryngeal surgery group. NFL levels 24 hours and seven days post-surgery were significantly increased in the cardiac group compared to the corresponding time points in the otolaryngeal group (mean difference, $174 \%, \mathrm{P}=0.01$, and mean difference, $1065 \%, \mathrm{P}<0.0001$, respectively; Figure 1B).

\section{$\mathbf{A} \boldsymbol{\beta} 40$}

A $\beta 40$ levels were significantly elevated during surgery compared to baseline (mean diff., $64.18 \mathrm{pg} / \mathrm{mL} ; \mathrm{P}<0.0001$ ), 24 hours post-surgery (mean diff., $34.57 \mathrm{pg} / \mathrm{mL} ; \mathrm{P}=0.0001$ ), and seven days post-surgery (mean diff., $36.96 \mathrm{pg} / \mathrm{mL} ; \mathrm{P}=0.0001$ ) in the cardiac group (Figure 1C). Also, A $\beta 40$ levels in the cardiac group at the end of surgery were significantly elevated compared to baseline (mean diff., $56.75 \mathrm{pg} / \mathrm{mL} ; \mathrm{P}<0.0001$ ), 24 hours post-surgery (mean diff., $27.15 \mathrm{pg} / \mathrm{mL} ; \mathrm{P}=0.005$ ), and seven days post-surgery (mean diff., $29.53 \mathrm{pg} / \mathrm{mL} ; \mathrm{P}=0.004$; Figure 1C). Similarly, A $\beta 40$ levels in the cardiac group remained elevated seven days postsurgery compared to baseline (mean diff., $27.22 \mathrm{pg} / \mathrm{mL} ; \mathrm{P}=0.01$ ). Interestingly, we found qualitatively similar results in the otolaryngeal group, except that $A \beta 40$ levels seven days post-surgery returned to baseline levels.

We also found that $A \beta 40$ levels after induced hypotension were significantly elevated in the cardiac group compared to the otolaryngeal group (mean diff., $27.79 \mathrm{pg} / \mathrm{mL} ; \mathrm{P}=0.02$; Figure $1 \mathrm{C})$.

\section{A $\beta 42$}

A 342 levels during surgery were elevated compared to baseline (mean diff., $59.3 \mathrm{pg} / \mathrm{mL}$; $\mathrm{P}=0.0001$ ), and 24 hours post-surgery (mean diff., $32.6 \mathrm{pg} / \mathrm{mL} ; \mathrm{P}=0.04$ ) in the cardiac group (Figure 1D). The A $\beta 42$ levels were also elevated at the end of surgery compared to baseline 
(mean diff., $79.8 \mathrm{pg} / \mathrm{mL}$; $\mathrm{P}<0.0001$ ), 24 hours post-surgery (mean diff., $53.0 \mathrm{pg} / \mathrm{mL}$; $\mathrm{P}=0.0001$ ), and seven days post-surgery (mean diff., $39.6 \mathrm{pg} / \mathrm{mL} ; \mathrm{P}=0.01$ ) in the cardiac group (Figure 1D). As with A $\beta 40$, changes in A $\beta 42$ levels in the otolaryngeal group followed a similar profile to the cardiac group between time points. Furthermore, $A \beta 42$ levels at the end of surgery were significantly elevated in the cardiac group compared to otolaryngeal group (mean diff., $44.1 \mathrm{pg} / \mathrm{mL} ; \mathrm{P}=0.04$; Figure 1C).

\section{Extracorporeal circulation and neuronal protein levels}

Of the 25 patients undergoing cardiac surgery, 16 were on extracorporeal circulation (onpump) and 9 underwent off-pump coronary artery bypass. On-pump cardiac surgery patients had elevated tau levels compared to off-pump cardiac surgery patients during surgery (mean difference, 2099\%; $\mathrm{P}=0.02$ ) and at the end of surgery (mean difference, 2213\%; $\mathrm{P}=0.01$, Figure 2A). The within-group profile of NFL change over time was similar for both on-pump and off-pump cardiac surgery patients. However, NFL levels for those on-pump were increased compared to those off-pump seven days post-surgery (mean difference, $431 \%$; $\mathrm{P}=0.006$; Figure 2B).

Furthermore, after comparing neuronal protein levels between the on-pump cardiac surgery group and the otolaryngeal surgery group, tau levels were found to be significantly elevated in the on-pump surgery group, particularly during surgery (mean difference, 2362\%; $\mathrm{P}<0.0001$ ), at the end of surgery (mean difference, 2453\%; $\mathrm{P}<0.0001$ ), and 24 hours post-surgery (mean difference, $1227 \% ; \mathrm{P}=0.009)$. Similarly, NFL levels were elevated in the on-pump cardiac surgery group compared to the otolaryngeal surgery group 24 hours post-surgery (mean difference, 202\%, $\mathrm{P}=0.009$ ) and seven days post-surgery (mean difference, 1184\%; $\mathrm{P}<0.0001)$. Differences were also analysed between the off-pump cardiac surgery group and the otolaryngeal surgery group. Tau levels were still elevated in the off-pump cardiac surgery group compared to the otolaryngeal surgery group during surgery (mean difference, $136 \%$; 
$\mathrm{P}=0.009$ ), at the end of surgery (mean difference, $152 \% ; \mathrm{P}=0.003$ ), and 24 hours post-surgery (mean difference, 138\%; P=0.006; Figure 3A). Similarly, NFL levels seven days post-surgery were still significantly elevated in the off-pump cardiac surgery group compared to the otolaryngeal surgery group (mean difference, 716\%; $\mathrm{P}<0.0001$; Figure 3B).

\section{No effect of myocardial infarction on neuronal markers}

To rule out that the increases in the neuronal biomarkers tau and NFL were due to release from the myocardium, these proteins were measured in blood samples from patients with myocardial infarction. However, myocardial infarction was not associated with increased release of tau as there was no correlation between plasma levels of troponin $\mathrm{T}$ and tau (rho=0.08, $\mathrm{P}=0.78$ ); nor was there any significant correlation between plasma levels of troponin $\mathrm{T}$ and NFL following myocardial infarction ( $\mathrm{rho}=0.50, \mathrm{P}=0.07)$.

\section{Discussion}

Patients undergoing cardiac surgery had markedly increased plasma levels of the neuronal protein tau during surgery and at the end of surgery compared to baseline levels in the same patient, as well as compared to the same time points in patients undergoing otolaryngeal surgery. Furthermore, patients undergoing cardiac surgery also had greatly increased plasma levels of NFL one week post-surgery, which was not observed in patients undergoing otolaryngeal surgery. In addition, extracorporeal circulation worsened the increase in plasma levels of both tau and NFL within the group of cardiac surgery patients.

Our findings are in concert with a previous study showing minor to moderate increases in tau and NFL in patients undergoing surgery [18]. However, that study examined a mixed population of mainly orthopaedic patients, with data presented at the group level and only a minority ( 6 out of 30 ) being cardiac surgery patients. Indeed, the changes were also much less pronounced than in the present study (250\% vs $1750 \%$ for tau and $67 \%$ vs $1100 \%$ for NFL). 
Importantly, no time-points after 48 hours were included in that study, meaning that any NFL peak around 7-10 days may have been overseen given the slow temporal course for the increase in blood NFL levels after an acute brain insult [21].

The most likely interpretation of our findings is that neuronal injury occurs to a greater extent in patients undergoing cardiac surgery than otolaryngeal surgery. It is foreseeable that an increase in tau could be due to a transient insult which in some cases may be reversible since these levels normalize after one week post-surgery. In contrast, since NFL is a part of neurofilaments, that are the main intermediary filament in axons, it is unlikely that the marked release of NFL reflects anything but neuronal damage. Supporting this are findings from neurodegenerative diseases where the highest levels of NFL are found in amyotrophic lateral sclerosis (ALS) where motor neurons with the longest axons in the body are irreversibly lost [22]. Increased levels of NFL have also been found in multiple sclerosis (MS) and they correlate with brain volume reduction and the number of contrasting enhanced lesions on MRI [23]. Further support of increased tau and NFL being related to the severity of neuronal damage comes from several studies that have shown marked increases in blood concentrations of these biomarkers after acute traumatic brain injury (TBI), with manifold higher levels in severe than in mild TBI, where higher levels predict functional and neurological outcomes $[21,24]$. Similarly, marked increases in tau and NFL are also found in patients with cardiac arrest, where higher levels are highly predictive of poor neurological outcome and survival $[25,26]$. Last, the time-course for the increase in tau and NFL was clearly different, with a rapid increase in plasma tau (peaking during the surgical procedure) but a slow gradual increase in NFL (with the highest levels one week post-surgery). This difference in temporal profile between tau and NFL is in agreement with previous studies $[18,21]$. The reason behind the difference in the temporal profile of tau and NFL is unknown but likely caused by different mechanisms where the release of tau may be caused by stress to neurons and active 
secretion, whereas the delayed release of NFL may occur when the neurons, and especially the axons, are damaged and neurofilaments are broken down slowly and released passively. Together with the lack of change in plasma $A \beta$ levels, the tau and NFL results suggest that these changes are not due to unspecific release of neurological proteins during or after general anaesthesia and surgery. Thus, our results indicate that cardiac surgery results in neuronal damage.

Why does neuronal damage occur to a greater extent in cardiac than otolaryngeal surgery? Mean arterial pressure was kept at $60-70 \mathrm{~mm} \mathrm{Hg}$ throughout both forms of surgeries in the present study ruling out hypoperfusion as a reason for the differences in tau and NFL levels. Extracorporeal circulation has been proposed as a cause of confusion, POCD and CNS damage in cardiac surgery patients $[27,28]$. In our study, the majority of cardiac surgery patients $(n=16)$ were on-pump, while a minority were off-pump ( $n=9)$. Indeed, plasma levels of tau were greatly elevated during surgery and at the end of surgery, as were NFL levels seven days post-surgery in the patients on-pump compared to those off-pump. Thus, our data indicate that extracorporeal circulation aggravates the neuronal damage caused by cardiac surgery.

We did not perform any a priori power calculation since this is the first study of its kind and therefore the expected effect size was unclear.

Neither tau nor NFL are expressed in the heart according to the expression database BioGPS (http://biogps.org). Thus, the increases in tau and NFL were not caused by direct damage to the heart during cardiac surgery but rather reflect neuronal damage. This conclusion was further supported by the lack of correlation between plasma levels of tau and NFL with plasma levels of troponin $\mathrm{T}$ in patients with myocardial infarction. 
Both tau and NFL levels are almost ten-fold higher in CSF compared to serum/plasma levels suggesting that the neuronal damage may be underestimated by studying serum levels [21, 29].

Regarding the difference in length of surgery between the otolaryngeal and cardiac groups, we cannot rule out that this may have affected the results. However, within both the cardiac and otolaryngeal groups, no association between tau or NFL levels and length of surgery was observed (data not shown), suggesting that the effect was minor.

Since tau and NFL are indicative of neuronal damage and death, future studies may analyse these biomarkers when developing new surgical methods to identify regimes with minimal effects on the CNS, aiming for reducing post-operative cognitive decline especially in elderly patients.

In conclusion, cardiac surgery was associated with a marked release of the neuronal markers tau during and at the end of surgery and NFL seven days post-surgery. This is likely indicative of neuronal damage, which was not observed in the contrast group of patients undergoing otolaryngeal surgery. Importantly, the increases in levels of these biomarkers reflecting neuronal were further aggravated by extracorporeal circulation. Thus, analyses of NFL and tau may in the future help to improve surgical procedures to minimize neuronal damage.

\section{Acknowledgements}

This study was funded by grants no. N/ST/ZB/14/001/1128, 143-28594L, 133-28785L, N/ST/ZB/16/001/1128 from Medical University of Bialystok Clinical Hospital, Poland, the Swedish Research Council, Swedish State Support for Clinical Research, Alzheimer's Association, the Knut and Alice Wallenberg Foundation, the Torsten Söderberg Foundation, the Alzheimer Foundation (Sweden), the European Research Council (grant number 681712), 
the Innovative Medicines Initiative Joint Undertaking under EMIF grant agreement $\mathrm{n}^{\circ}$ 115372, resources of which are composed of financial contribution from the European Union's Seventh Framework Program (FP7/2007-2013) and EFPIA companies.

\section{Disclosure}

PL received consultation and/or lecture honoraria from IBL International, Fujirebio Europe, AJ Roboscreen, and Roche. KB has served as a consultant or at advisory boards for Alzheon, BioArctic, Biogen, Eli Lilly, Fujirebio Europe, IBL International, Merck, Novartis, Pfizer and Roche Diagnostics. HZ has served on scientific advisory boards for Roche Diagnostics, Wave, Samumed and CogRx. None of the other authors declares competing interests. 
Table 1 Cardiac surgery patients

\begin{tabular}{lllll}
\hline ID & Procedure & ECT & Age & Sex \\
\hline 1 & MVI & 128 & 59 & $\mathrm{~F}$ \\
2 & AVR, MVR & 166 & 77 & $\mathrm{~F}$ \\
3 & OPCAB & NA & 63 & $\mathrm{M}$ \\
4 & AVR & 89 & 64 & $\mathrm{M}$ \\
5 & AVR & 127 & 61 & $\mathrm{~F}$ \\
6 & OPCAB & NA & 62 & $\mathrm{M}$ \\
7 & AVR, MVR, TVP & 185 & 70 & $\mathrm{~F}$ \\
8 & Bentall & 109 & 76 & $\mathrm{~F}$ \\
9 & OPCAB & NA & 80 & $\mathrm{M}$ \\
10 & CABG & 102 & 72 & $\mathrm{M}$ \\
11 & OPCAB & NA & 76 & $\mathrm{M}$ \\
12 & AVR, MVR, CABG & 196 & 79 & $\mathrm{M}$ \\
13 & AVR, MVR & 181 & 70 & $\mathrm{M}$ \\
14 & MVR & 190 & 45 & $\mathrm{M}$ \\
15 & OPCAB & NA & 70 & $\mathrm{~F}$ \\
16 & CABG, SVR & 70 & 52 & $\mathrm{~F}$ \\
17 & MVR, TVP & 192 & 58 & $\mathrm{~F}$ \\
18 & CABG & 89 & 67 & $\mathrm{M}$ \\
19 & CABG & 130 & 67 & $\mathrm{~F}$ \\
20 & CABG & 85 & 76 & $\mathrm{M}$ \\
21 & CABG & 101 & 80 & $\mathrm{~F}$ \\
22 & OPCAB & NA & 60 & M \\
23 & OPCAB & NA & 62 & F \\
24 & OPCAB & NA & 68 & M \\
25 & OPCAB & NA & 66 & F \\
\hline
\end{tabular}

ECT: extracorporeal circulation time, MVI: mitral valve implantation, AVR: aortic valve replacement, MVR: mitral valve replacement, OPCAB:

off pump coronary artery bypass, TVP: tricuspid valve procedure, SVR: surgical ventrical restoration, CABG: coronary artery bypass grafting. 
Table 2 Otolaryngeal surgery patients

\begin{tabular}{lllll}
\hline ID & Procedure & ECT & Age & Sex \\
\hline 1 & FESS, SS & NA & 51 & F \\
2 & FESS & NA & 50 & F \\
3 & FESS & NA & 33 & M \\
4 & FESS, SS, INF, CP & NA & 34 & M \\
5 & FESS, EOD, SS & NA & 35 & F \\
6 & FESS, CP & NA & 43 & M \\
7 & FESS, SS, CP & NA & 31 & F \\
8 & FESS & NA & 31 & F \\
9 & FESS, SS & NA & 48 & M \\
10 & FESS & NA & 69 & M \\
11 & FESS & NA & 47 & M \\
12 & FESS & NA & 51 & M \\
13 & FESS & NA & 59 & M \\
14 & FESS, SS & NA & 55 & M \\
15 & FESS, SS, INF & NA & 25 & F \\
16 & FESS, SS & NA & 18 & F \\
17 & FESS & NA & 65 & F \\
18 & FESS, SS & NA & 63 & M \\
19 & FESS & NA & 25 & F \\
20 & FESS & NA & 51 & M \\
21 & EOD & NA & 56 & F \\
22 & FESS, SS & NA & 53 & M \\
23 & FESS & NA & 23 & F \\
24 & FESS & NA & 54 & M \\
25 & FESS & NA & 33 & F \\
26 & FESS & NA & 52 & F \\
\hline & & & & \\
\hline
\end{tabular}


ECT: extracorporeal circulation time, FESS: functional endoscopic sinus surgery, SS: sinus surgery (extended), INF: infundibulotomy, CP: conchoplasty, EOD: endoscopic orbital decompression. 


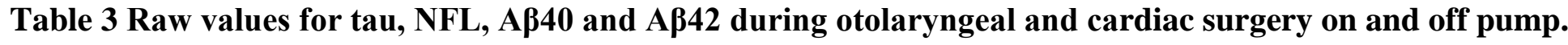

\begin{tabular}{|c|c|c|c|c|}
\hline Biomarker & Otolaryngeal surgery & Cardiac surgery combined & Cardiac surgery on-pump & Cardiac surgery off-pump \\
\hline Tau 1 & $2.8(1.6-4.6)$ & $3.2(2.3-4.1)$ & $3.2(2.4-4.1)$ & $3.1(2.3-3.9)$ \\
\hline Tau 2 & $3.2(2.1-6.4)$ & $16.4(11.3-51.6)$ & $33.9(15.9-60.3)$ & $7.2(6.5-60.3)$ \\
\hline Tau 3 & $3.5(2.6-4.8)$ & $21.8(8.8-50.5)$ & $47.1(23.4-60.6)$ & $7.9(7.1-9.0)$ \\
\hline Tau 4 & $2.0(1.6-3.0)$ & $6.6(5.0-11.2)$ & $8.3(6.4-13.2)$ & $4.7(3.6-6.8)$ \\
\hline Tau 5 & $1.7(1.6-2.2)$ & $3.1(2.4-5.4)$ & $3.1(2.4-5.0)$ & $2.9(2.5-6.2)$ \\
\hline NFL 1 & $9.0(7.0-13.1)$ & $21.2(13.4-35.7)$ & $19.3(12.9-27.5)$ & $29.5(16.5-54.8)$ \\
\hline NFL 2 & $9.2(6.9-17.2)$ & $22.8(13.4-34.9)$ & $20.5(13.4-30.7)$ & $29.5(19.0-65.0)$ \\
\hline NFL 3 & $11.4(8.0-20.6)$ & $25.5(20.4-38.4)$ & $28.3(21.3-36.1)$ & $22.4(17.7-43.8)$ \\
\hline NFL 4 & $10.6(8.2-21.5)$ & $40.9(26.6-57.6)$ & $41.8(33.1-57.4)$ & $38.1(22.0-83.1)$ \\
\hline NFL 5 & $16.4(12.6-25.1)$ & $188.8(154.3-288.4)$ & $188.8(166.4-299.4)$ & $219.3(142.2-284.4)$ \\
\hline $\mathrm{A} \beta 401$ & $114.9(104.0-147.9)$ & $194.9(175.2-221.9)$ & $192.8(178.4-225.0)$ & $201.2(149.1-204.9)$ \\
\hline $\mathrm{A} \beta 402$ & $137.6(126.0-162.1)$ & $214.3(174.9-244.6)$ & $184.8(168.5-229.8)$ & $238.3(220.5-308.3)$ \\
\hline $\mathrm{A} \beta 403$ & $137.9(113.0-153.6)$ & $192.4(153.8-232.2)$ & $184.1(153.0-219.6)$ & $202.4(169.2-232.2)$ \\
\hline $\mathrm{A} \beta 404$ & $123.7(105.7-132.5)$ & $184.3(161.7-253.6)$ & $185.0(172.6-255.2)$ & $184.3(153.5-253.6)$ \\
\hline $\mathrm{A} \beta 405$ & $125.7(109.1-141.0)$ & $196.4(174.6-223.2)$ & $196.9(179.1-250.3)$ & $167.7(148.7-216.2)$ \\
\hline $\mathrm{A} \beta 421$ & $36.0(29.6-41.9)$ & $43.3(35.7-52.7)$ & $45.2(36.1-55.9)$ & $39.3(35.7-42.3)$ \\
\hline $\mathrm{A} \beta 422$ & $45.4(37.2-49.6)$ & $44.9(35.1-49.0)$ & $42.0(31.5-45.8))$ & $48.2(44.1-51.9)$ \\
\hline $\mathrm{A} \beta 423$ & $42.7(35.1-48.0)$ & $44.1(39.2-51.2)$ & $40.6(35.2-51.5)$ & $45.3(41.9-50.0)$ \\
\hline $\mathrm{A} \beta 424$ & $37.2(30.6-42.8)$ & $38.5(28.1-49.9)$ & $38.9(28.0-50.2)$ & $38.3(34.5-42.5)$ \\
\hline $\mathrm{A} \beta 425$ & $36.3(32.7-42.0)$ & $44.9(41.0-51.2)$ & $46.4(42.5-53.5)$ & $41.7(35.2-44.0)$ \\
\hline
\end{tabular}

Timepoints: 1 baseline, 2 during surgery, 3 at the end of surgery, 4 24-hours post-surgery and 5 seven days post-surgery. Data are presented as

median and interquartile range. 


\section{References}

[1\} 489-498.

$[2\}$

Gilman S (1965) Cerebral Disorders after Open-Heart Operations. N Engl J Med 272, abnorn Javid H, Tufo HM, Najafi H, Dye WS, Hunter JA, Julian OC (1969) Neurological malities following open-heart surgery. J Thorac Cardiovasc Surg 58, 502-509.

[3\} Moller JT, Cluitmans P, Rasmussen LS, Houx P, Rasmussen H, Canet J, Rabbitt P, Jolles J, Larsen K, Hanning CD, Langeron O, Johnson T, Lauven PM, Kristensen PA, Biedler A, van Beem H, Fraidakis O, Silverstein JH, Beneken JE, Gravenstein JS (1998) Long-term postoperative cognitive dysfunction in the elderly ISPOCD1 study. ISPOCD investigators. International Study of Post-Operative Cognitive Dysfunction. Lancet 351, 857-861.

[4\} Newman MF, Kirchner JL, Phillips-Bute B, Gaver V, Grocott H, Jones RH, Mark DB, Reves JG, Blumenthal JA, Neurological Outcome Research G, the Cardiothoracic Anesthesiology Research Endeavors I (2001) Longitudinal assessment of neurocognitive function after coronary-artery bypass surgery. $N$ Engl J Med 344, 395-402.

[5] Scott DA, Evered LA, Gerraty RP, MacIsaac A, Lai-Kwon J, Silbert BS (2014) Cognitive dysfunction follows left heart catheterisation but is not related to microembolic count. Int $J$ Cardiol 175, 67-71.

[6] van Harten AE, Scheeren TW, Absalom AR (2012) A review of postoperative cognitive dysfunction and neuroinflammation associated with cardiac surgery and anaesthesia. Anaesthesia 67, 280-293.

[7] Mason SE, Noel-Storr A, Ritchie CW (2010) The impact of general and regional anesthesia on the incidence of post-operative cognitive dysfunction and post-operative delirium: a systematic review with meta-analysis. J Alzheimers Dis 22 Suppl 3, 67-79.

[8\} Rasmussen LS, Johnson T, Kuipers HM, Kristensen D, Siersma VD, Vila P, Jolles J, Papaioannou A, Abildstrom H, Silverstein JH, Bonal JA, Raeder J, Nielsen IK, Korttila K, Munoz L, Dodds C, Hanning CD, Moller JT, Investigators I (2003) Does anaesthesia cause postoperative cognitive dysfunction? A randomised study of regional versus general anaesthesia in 438 elderly patients. Acta Anaesthesiol Scand 47, 260-266.

[9] Williams-Russo P, Sharrock NE, Mattis S, Szatrowski TP, Charlson ME (1995) Cognitive effects after epidural vs general anesthesia in older adults. A randomized trial. JAMA 274, 44-50.

[10\} Basile AM, Fusi C, Conti AA, Paniccia R, Trefoloni G, Pracucci G, Di Carlo A, Noferi D, Carbonetto F, Pretelli P, Calamai G, Vaccari M, Abbate R, Inzitari D (2001) S-100 protein and neuron-specific enolase as markers of subclinical cerebral damage after cardiac surgery: preliminary observation of a 6-month follow-up study. Eur Neurol 45, 151-159.

[11\} Zetterberg H, Blennow K (2016) Fluid biomarkers for mild traumatic brain injury and related conditions. Nat Rev Neurol 12, 563-574.

[12\} Olsson B, Lautner R, Andreasson U, Ohrfelt A, Portelius E, Bjerke M, Holtta M, Rosen C, Olsson C, Strobel G, Wu E, Dakin K, Petzold M, Blennow K, Zetterberg H (2016) CSF and blood biomarkers for the diagnosis of Alzheimer's disease: a systematic review and meta-analysis. Lancet Neurol 15, 673-684.

[13\} Ost M, Nylen K, Csajbok L, Ohrfelt AO, Tullberg M, Wikkelso C, Nellgard P, Rosengren L, Blennow K, Nellgard B (2006) Initial CSF total tau correlates with 1-year outcome in patients with traumatic brain injury. Neurology 67, 1600-1604.

[14\} Yamasaki H, Itakura C, Mizutani M (1991) Hereditary hypotrophic axonopathy with neurofilament deficiency in a mutant strain of the Japanese quail. Acta Neuropathol 82, 427-434.

[15 \} Zetterberg H (2016) Neurofilament Light: A Dynamic Cross-Disease Fluid Biomarker for Neurodegeneration. Neuron 91, 1-3.

[16\} Gisslen M, Price RW, Andreasson U, Norgren N, Nilsson S, Hagberg L, Fuchs D, Spudich S, Blennow K, Zetterberg H (2016) Plasma Concentration of the Neurofilament Light Protein (NFL) is a Biomarker of CNS Injury in HIV Infection: A Cross-Sectional Study. EBioMedicine 3, 135-140. 
[17\} Randall J, Mortberg E, Provuncher GK, Fournier DR, Duffy DC, Rubertsson S, Blennow K, Zetterberg H, Wilson DH (2013) Tau proteins in serum predict neurological outcome after hypoxic brain injury from cardiac arrest: results of a pilot study. Resuscitation 84, 351-356. [18\} Evered L, Silbert B, Scott DA, Zetterberg H, Blennow K (2018) Association of Changes in Plasma Neurofilament Light and Tau Levels With Anesthesia and Surgery: Results From the CAPACITY and ARCADIAN Studies. JAMA Neurol 75, 542-547.

[19\} Blennow K, Mattsson N, Scholl M, Hansson O, Zetterberg H (2015) Amyloid biomarkers in Alzheimer's disease. Trends Pharmacol Sci 36, 297-309.

[20] Calafiore AM, Teodori G, Mezzetti A, Bosco G, Verna AM, Di Giammarco G, Lapenna D (1995) Intermittent antegrade warm blood cardioplegia. Ann Thorac Surg 59, 398-402. [21] Shahim P, Gren M, Liman V, Andreasson U, Norgren N, Tegner Y, Mattsson N, Andreasen N, Ost M, Zetterberg H, Nellgard B, Blennow K (2016) Serum neurofilament light protein predicts clinical outcome in traumatic brain injury. Sci Rep 6, 36791.

[22\} Feneberg E, Oeckl P, Steinacker P, Verde F, Barro C, Van Damme P, Gray E, Grosskreutz J, Jardel C, Kuhle J, Koerner S, Lamari F, Amador MDM, Mayer B, Morelli C, Muckova P, Petri S, Poesen K, Raaphorst J, Salachas F, Silani V, Stubendorff B, Turner MR, Verbeek MM, Weishaupt JH, Weydt P, Ludolph AC, Otto M (2018) Multicenter evaluation of neurofilaments in early symptom onset amyotrophic lateral sclerosis. Neurology 90, e22-e30.

[23\} Barro C, Benkert P, Disanto G, Tsagkas C, Amann M, Naegelin Y, Leppert D, Gobbi C, Granziera C, Yaldizli O, Michalak Z, Wuerfel J, Kappos L, Parmar K, Kuhle J (2018) Serum neurofilament as a predictor of disease worsening and brain and spinal cord atrophy in multiple sclerosis. Brain.

[24\} Bernick C, Zetterberg H, Shan G, Banks S, Blennow K (2018) Longitudinal Performance of Plasma Neurofilament Light and Tau in Professional Fighters: The Professional Fighters Brain Health Study. J Neurotrauma 35, 2351-2356.

[25] Mattsson N, Zetterberg H, Nielsen N, Blennow K, Dankiewicz J, Friberg H, Lilja G, Insel PS, Rylander C, Stammet P, Aneman A, Hassager C, Kjaergaard J, Kuiper M, Pellis T, Wetterslev J, Wise M, Cronberg T (2017) Serum tau and neurological outcome in cardiac arrest. Ann Neurol 82, 665-675.

[26\} Rosen C, Rosen H, Andreasson U, Bremell D, Bremler R, Hagberg L, Rosengren L, Blennow K, Zetterberg H (2014) Cerebrospinal fluid biomarkers in cardiac arrest survivors. Resuscitation 85, 227-232.

[27\} Al-Ruzzeh S, George S, Bustami M, Wray J, Ilsley C, Athanasiou T, Amrani M (2006) Effect of off-pump coronary artery bypass surgery on clinical, angiographic, neurocognitive, and quality of life outcomes: randomised controlled trial. BMJ 332, 1365.

[28\} Van Dijk D, Jansen EW, Hijman R, Nierich AP, Diephuis JC, Moons KG, Lahpor JR, Borst C, Keizer AM, Nathoe HM, Grobbee DE, De Jaegere PP, Kalkman CJ, Octopus Study G (2002) Cognitive outcome after off-pump and on-pump coronary artery bypass graft surgery: a randomized trial. JAMA 287, 1405-1412.

[29\} Mattsson N, Zetterberg H, Janelidze S, Insel PS, Andreasson U, Stomrud E, Palmqvist S, Baker D, Tan Hehir CA, Jeromin A, Hanlon D, Song L, Shaw LM, Trojanowski JQ, Weiner MW, Hansson O, Blennow K, Investigators A (2016) Plasma tau in Alzheimer disease. Neurology 87, 18271835.

\section{Figure legends}

Figure 1. Plasma levels of tau, NFL, Aß40 and Aß42 before, during and after cardiac

and otolaryngeal surgery. (A) Changes in plasma levels of tau, (B) NFL, (C) Aß40 and (D) 
A $\beta 42$ before, during and after cardiac surgery and otolaryngeal surgery. The levels are normalized in each figure and the baseline is set to $100 \%$. The P-values are adjusted for age and sex whereas the plasma levels in the graphs are the raw data values. Data are presented as mean \pm SEM. Baseline (sample 1), during surgery (sample 2), at the end of surgery (sample 3), 24 hours post-surgery (sample 4), and 7 days post-surgery (sample 5).

Figure 2. Plasma levels of tau and NFL before, during and after cardiac surgery on or off pump. (A) Tau and (B) NFL before, during and after cardiac surgery on or off extracorporeal circulation. The levels are normalized in each figure and the baseline is set to $100 \%$. The P-values are adjusted for age and sex whereas the plasma levels in the graphs are the raw data values. Data are presented as mean \pm SEM. Baseline (sample 1), during surgery (sample 2), at the end of surgery (sample 3), 24 hours post-surgery (sample 4), and 7 days post-surgery (sample 5).

Figure 3. Plasma levels of tau and NFL before, during and after cardiac surgery off-pump and otolaryngeal surgery. (A) Tau and (B) NFL before, during and after cardiac surgery off extracorporeal circulation and otolaryngeal surgery. The P-values are adjusted for age and sex whereas the plasma levels in the graphs are the raw data values. The levels are normalized in each figure and the baseline is set to $100 \%$. Data are presented as mean \pm SEM. Baseline (sample 1), during surgery (sample 2), at the end of surgery (sample 3), 24 hours post-surgery (sample 4), and 7 days post-surgery (sample 5). 


\section{Figures}

\section{Figure 1}
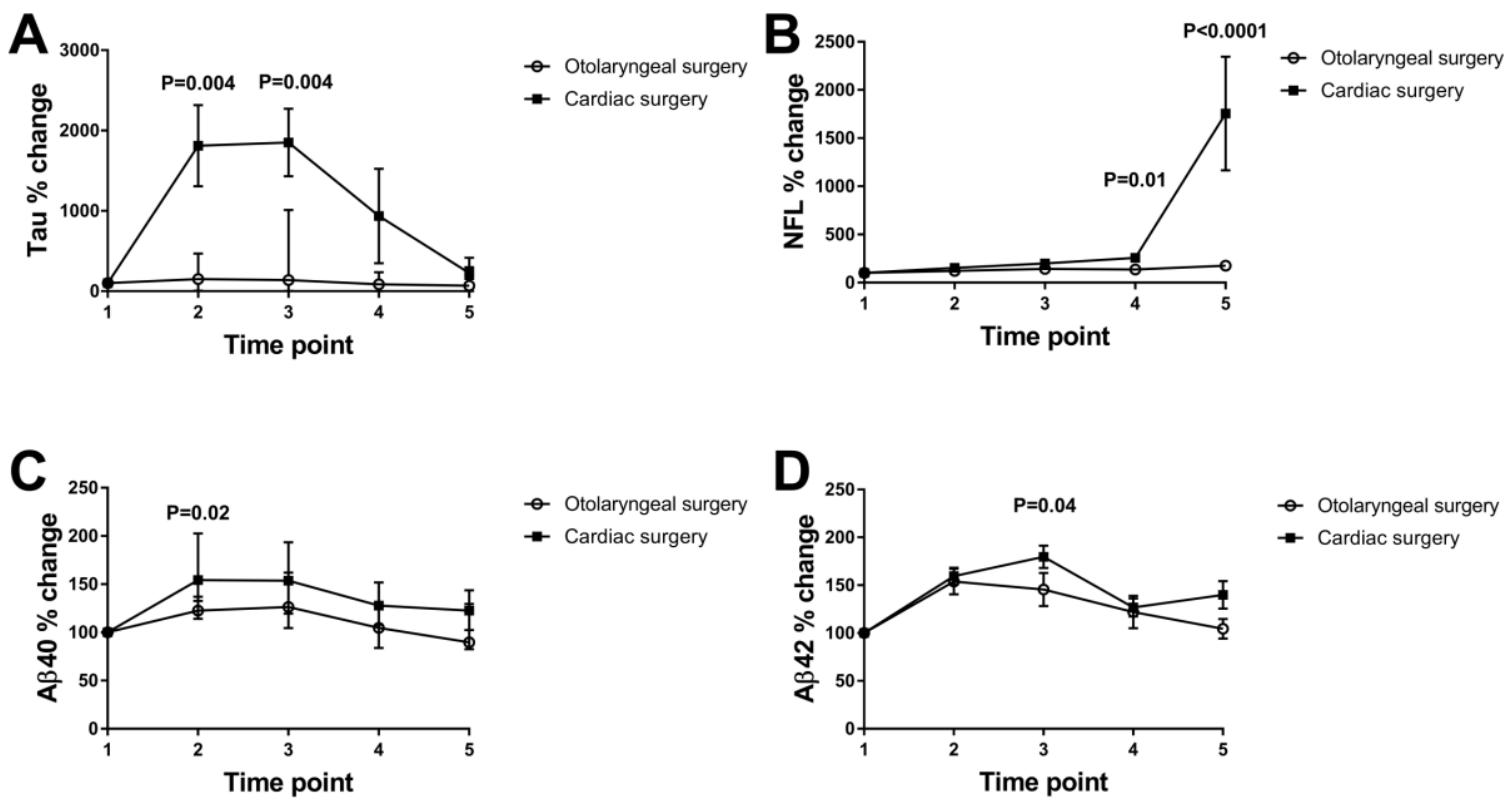

\section{Figure 2}
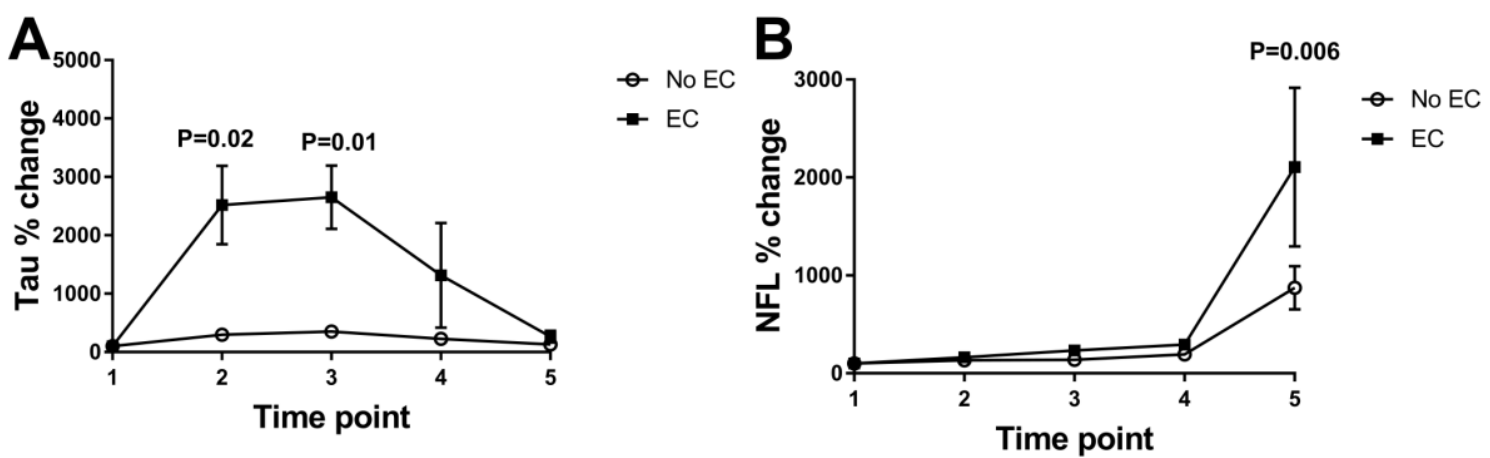

\section{Figure 3}
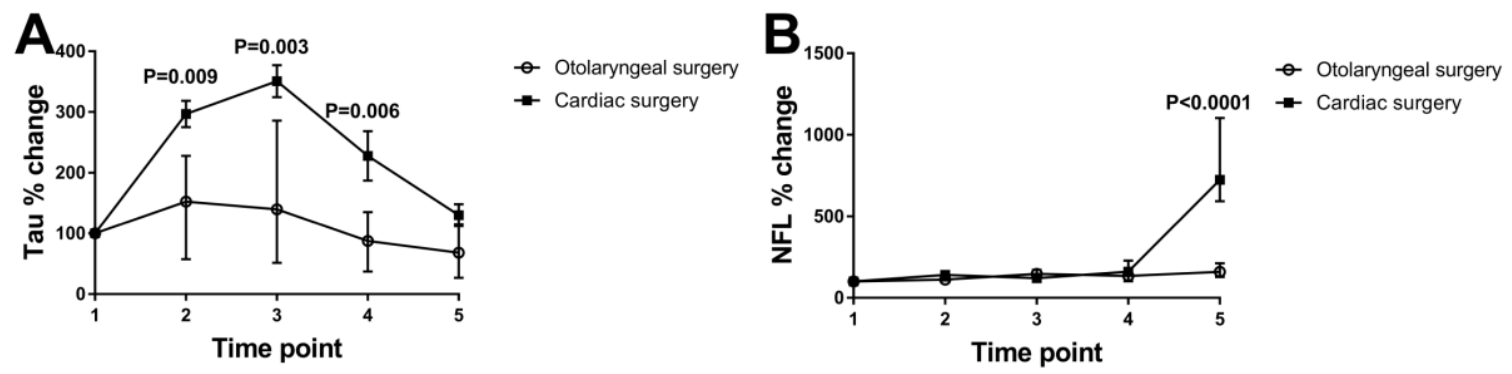